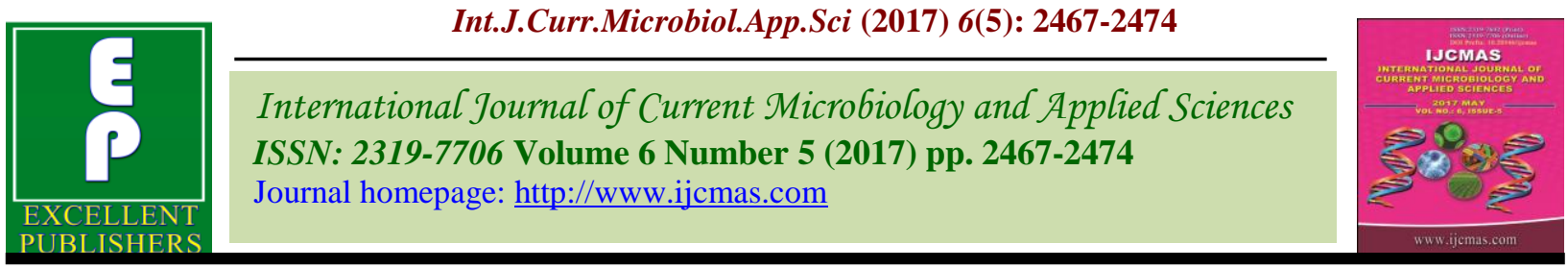

Review Article

https://doi.org/10.20546/ijcmas.2017.605.276

\title{
Defense Induced by Jasmonic Acid: A Review
}

\author{
M.K. Samota ${ }^{1}$, L. Bhatt ${ }^{2}$, N. Garg ${ }^{1}$ and N. Geat ${ }^{1}$ \\ ${ }^{1}$ Division of Biochemistry, IARI, New Delhi-110012, India \\ ${ }^{2}$ G.B. Pant University of Agriculture and Technology, Pantnagar- 263145, India \\ *Corresponding author
}

\begin{abstract}
A B S T R A C T
A number of novel JA derivatives have been synthesized, isolated, and characterized. A clearer picture of jasmonate biosynthesis and functions has emerged that in many respects

Keywords resembles the synthesis and functions of prostaglandins and leukotrienes in mammalians. Jasmonates are produced from a-linolenic acid from lipids of damaged plant membranes. The biosynthesis of these signalling compounds can be triggered through the activation of

Jasmonic acid, a-linolenic acid, Leukotrienes, Pharmacological agents

Article Info

Accepted:

25 April 2017 Available Online: 10 May 2017 cell membrane receptors by pathogens, peptide signals, or wounding. These jasmonates are widely distributed in plants and affect a various processes, including fruit ripening, production of viable pollen, root growth, and plant responses to wounding, abiotic stress, infections, and insects. Many of these new compounds have potent biological activity, promising that novel types of pharmacological agents possessing new mechanisms of action could soon become available. All these effects of JA are indirect, due to the wellstudied induction of the biosynthesis of secondary compounds like anthocyanins, glucosinolates, terpenoids, or phenolics via transcription factors such as the Myb, Myc, and ORCA families. JA and its derivatives are widely use in daliy life as medicinal, cosmetic, and flavoring uses and other applications in agriculture systems. During the last 20 years, the many of research and inventions have showed that JA, (p)-7-iso-JA and MJ have anticancer activity against various cancers (e.g., prostate, lymphoid, breast, blood, skin cancers, etc.).
\end{abstract}

\section{Introduction}

Plants are very delicate have no opportunity other than to adapt their growth and changing with environment and need to coordinate their growth and physiology responses. Plants present in an environment in ancient time characterized by the presence of various types of stresses both biotic and abiotic. Phytohormones play important role in plant growth, development and response to stresses. Plant hormones regulate diverse developmental processes and send the signals networks in plants under different abiotic stresses. With the advancement in plant biology research it has been established that phytohormones have the potential to remove the deleterious effects posed by abiotic stresses (Masood et al., 2012; Khan et al., 2013). Plant defense responses are mediated by three major pathways of the phytohormones, jasmonic acid (JA), salicylic acid (SA) and ethylene (ET). The fine tuning of plant response to pathogen depends on the synergistic or antagonistic interactions between these three pathways (Takahashi et al., 2004). According to (Creelman and Mulpuri, 2002; Wasternack and Hause, 2002; 
Wasternack, 2014 Jasmonates widely role in cellular regulators involved in diverse developmental processes such as seed germination, root growth, gravitropism, trichome formation, embryo development, sex determination (maize), fertility, seedling development, tuber formation, leaf movement, fruit ripening and leaf senescence Jasmonates regulate a wide range of plant processes, such as growth and development, including defense against biotic and abiotic stresses (Browse and Howe, 2008; Reinbothe et al., 2009). Wasternack (2007) and Gfeller et al., (2010) studied that the derivatives of alinolenic acid viz. jasmonic acid and jasmonoyl-L-isoleucine (JAIle) are accumulate in plants in response to biotic and abiotic stress. Xue and Zhang (2007) studied that Jasmonic acid regulate the leaf and root morphogenesis in soybean. Jasmonic acid was first of all isolated from the fungal cultures of Lasiodiplodiatheobromae while as methyl jasmonate (MeJA; Fig. 1) was obtained from the essential oil of Jasminumgrandiforum L. And Rosmarinus officinalis L. Jasmonates are present in all higher plants and the level is higher in flowers and reproductive tissues, while low in roots and mature leaves. The derivatives of jasmonic acid are various types and mainly hydroxylated forms like tuberonic acid and cucurbic acid and other amino acid conjugates (Hamberg and Gardner, 1992). Koda (1992) revealed that tuberonic acid (12hydroxy-JA) and its glucoside regulate the tuber formation in potato; however, recent study has indicated that the process is controlled by light, temperature and gibberellic acid (Lin et al., 2013). Therefore, itwas concluded that the JAs control tuber formation indirectly through crosstalk with GA signaling (Wasternack and Hause, 2013). Recently Michelet et al., 2012 found that one of the derivatives of the jasmonic acid, namely tetra-hydro jasmonic acid (LR2412) has shown anti-aging potential in case of human skin. LR2412 is known to increase the expression of hyaluronase synthase 2 (HAS2) and hyaluronase synthase 3 (HAS3) which consequently increase the synthesis of hyaluronic acid (HA) Plants are associated with diverse microbial communities that influence their health and nutrition. These organisms are known as the plant microbiome and used as more sustainably maintain or enhance global food security. To achieve this, ways to manipulate the structure of plantassociated microbial communities need to be identified. Recently, activation of the jasmonic acid (JA) plant defence pathway, that are involved in suppression of necrotrophic pathogens and herbivorous insects, was shown to alter the composition of the Arabidopsis thaliana root microbiome. Activation of the JA give signal to pathway increased the relative abundances of bacterial populations closely related to taxa that are reported to suppress phytopathogens and insects. This suggests that when these attack the plants may have evolved mechanisms to recruit symbionts that enhance their tolerance to biotic stress. Currently, however, it is not found whether the microbiomes of other plant species are influenced by activation of the JA pathway, and whether these effects, if any, are also present in endophytic compartments of the host. Given the intimate physical association between plants and endophytic symbionts, changes to the structure of endophytic communities may disproportionately influence host fitness. While JA give the signal has been shown to restrict endophytic colonisation of rice (Oryza sativa) by incompatible strains of nitrogenfixing Azoarcusbacteria and suppress nodulation in Lotus japonica it remains unknown whether JA gives the signal influences the overall structure of endophytic microbiomes. Wheat is one of the most important and widely grown crops worldwide. Despite this, the effects of JA signalling on wheat microbial communities have not been characterised. In this research, we used 
phylogenetic marker gene sequencing to determine whether activation of the JA pathway altered the diversity of bacterial and archaeal communities associated with the wheat rhizosphere and root and shoot endophytic environments. Increased JA signalling was achieved via exogenous application of methyl jasmonate (MeJA) and confirmed by quantification of JA-associated gene transcripts. In the last year, we measured the total enzymatic activity and substrate utilisation profiles of microbial communities associated with the rhizosphere (Liu et al., 2016). Phytohormones play important roles in mediating host responses to various abiotic stresses. Increasing evidence has shown that jasmonic acid (JA, or the related compound methyl jasmonate, MeJA) functions in diverse physiological processes in plants, such as the regulation of plant responses to abiotic stresses.

The exogenous application of JA to wheat seedlings for three days has been shown to significantly enhance the salt tolerance of these plants by increasing the activities of antioxidant enzymes and the concentrations of antioxidative compounds. MeJA improves salinity resistance in German chamomile by increasing the activity of antioxidant enzymes. The endogenous JA content has been shown to be markedly increased under drought and cold stresses but reduced under heat stress in rice. Expression profiling and physiological characterization on salinity stress response in barley reveal the JAmediated adaptation to salinity stress. Zhao et $a l$, showed that a wheat allene oxide cyclase (AOC) gene enhances salinity tolerance via the JA signalling pathway8. The steady-state levels of two key enzymes in JA biosynthesis, lipoxygenase (LOX) and allene oxide synthase (AOS), and the content of JA are higher in salt-tolerant tomato cultivars9. The salt hypersensitivity of the lox 3 mutant can be complemented by MeJA10 (Zhang et al., 2009).

\section{Biosynthesis of jasmonate}

The higher amounts of B present in soil also decrease the net photosynthetic rate, closing of stomatal, internal $\mathrm{CO} 2$ concentration and total chlorophyll content in leaves (Aftab et al., 2011). B toxicity leads to the accumulation of reactive oxygen species (ROS) in wheat (Gunes et al., 2007), barley (Inal et al., 2009) and tomato (Cervilla et al., 2007).Paull et al., 1992; Noctar et al., 1997 found that when exposed to B stress include leaf burn, decreased fruit size and number, delayed development and reduced vigor The typical symptoms showed by plant.Exogenous application of MeJA has been shown to counter the boron toxicity in plants. When boron stressed plants of Artemisia were foliarly sprayed with MeJA, it trigger the synthesis of antioxidant enzymes, reduced the amount of lipid peroxidation and enhances artemisinin content.

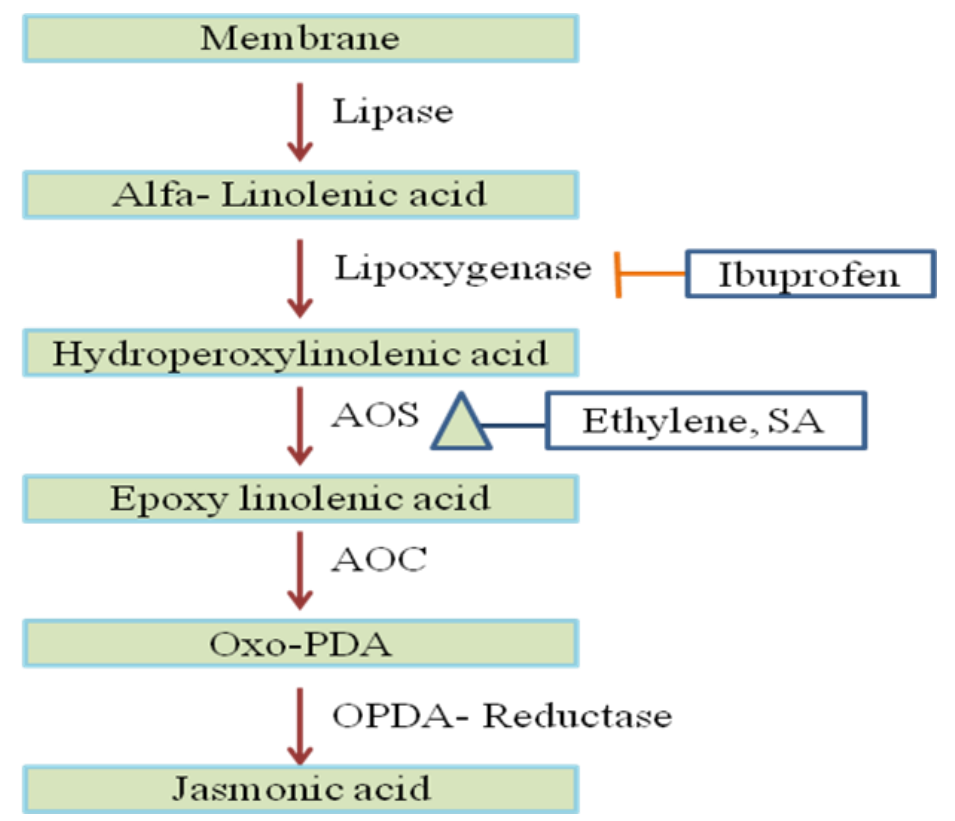

\section{Role of jasmonates in chilling stress}

Chilling injury $(\mathrm{Cl})$ creates storage problems for the horticultural crops, especially growing in the tropical and sub-tropical regions. The fruits of the tropical plants exhibit chilling 
injury symptoms, including browning of the skin, greater firmness in texture, and offflavor in the fruit. It has been found that MeJA treatment reduce the chilling injury in the fruits of plants like zucchini, mango, avocado, sweet pepper, tomato and papaya (Wang and Buta, 1994; Meir et al., 1998; Gonzalez-Aguilar et al., 2000, 2003). JAs induce the production of a diverse array of chemicals such as proteinase inhibitors, antimicrobial secondary compounds, antioxidants, pathogenesis-related and cellular protection molecules, including proteins involved in detoxification and redox balance (Farmer and Ryan, 1990; Farmer, 2007; Cao et al., 2009; Soares et al., 2010; Guo et al., 2013; Zhou et al., 2013; Gill et al., 2013). MeJA significantly application reduced the CI index and electrolyte leakage in pineapple. Browning discoloration mostly occurs by the accumulation of phenolics compounds and the polyphenol oxidase in the fruits (Paull and Rohrbach, 1985). However, when the fruits of pineapple were treated with MeJA, total phenolic content was significantly reduced as compared to the non-treated plants, which ultimately protects the fruits from the browning discoloration. It has been discoverd that MeJA treatment can reduce the development of chilling injury symptoms in zucchini (Wang and Buta, 1994) and mango (Gonzalez-Aguilar et al., 2000).

\section{Boron stress and role of jasmonate}

Boron (B) is an essential nutrient element growth and development of plants. However, in excess amount it may be toxic to the plants and can cause oxidative stress and considerably reduces the stem length, fresh and dry masses of the plants as in Artemisia annua. Aftab et al., 2011 studied that the excess amounts of $\mathrm{B}$ present in soil also decrease the net photosynthetic rate, closing of stomatal, internal $\mathrm{CO} 2$ concentration and total chlorophyll content in leaves. B toxicity leads to the accumulation of reactive oxygen species (ROS) in wheat (Gunes et al., 2007), barley (Inal et al., 2009) and tomato (Cervilla et al., 2007). The typical symptoms showed by plants when exposed to B stress include leaf burn, decreased fruit size and number, delayed development and reduced vigor (Paull et al., 1992; Noctor et al., 1998). Exogenous application of MeJA has been shown to counter the boron toxicity in plants. When boron stressed plants of Artemisia were foliarly sprayed with MeJA, it started to stimulate the synthesis of antioxidant enzymes, reduced the amount of lipid peroxidation and enhances artemisinin content.

\section{Anticancer activity}

JA and its derivatives, which are fatty acidderived cyclopentanones, occur widely occur in the plant kingdom and have known for various biological activities. One case study Halitschke et al., 2004 found that the first report on jasmonate-induced anticancer activities exhibited their capacity to cause both cell death and suppression of cell proliferation. Ferrari et al., 2003 found that Jasmonates are small hydrophobic compounds that act as plant stress hormones and can increase the survival of lymphomabearing animals and humans, and induce death in human leukemia, lung, colon, prostate, neuroblastoma, breast, and melanoma cell lines, and also effect in leukemic cells from chronic lymphocytic leukemia (CLL) patients. MJ was studied in topical application for precancerous and cancerous skin lesions (Pedranzani, et al., 2003). Accorandly (Reinbot et al., 2009) indicated that a JA derivative, 3-hydroxy2(S)-(2Z-butenyl)- cyclopentane-1(S)-acetic acid, obtained by biotransformation with the fungus Gibberellins fujikuroi, was able to inhibit the growth of both androgen-sensitive (LNCaP) and androgen in sensitive (DU-145) 
prostate cancer cells. The apoptosis evocated by this new molecule appeared to associated with an increase in reactive oxygen species (ROS) production (Reinbothe et al., 2009). A study indicated that three JA derivatives obtained by biotransformation were specifically active on several types of cancer cells (Carvajal et al., 2011, 2012, 2013).

\section{Anti-inflammatory activities}

The results of studies on the potential antiinflammatory effects of MJ derived from the red algaGracilariverrucosshowed that MJ was comparable to or more effective than prostaglandin-like compounds (Dang et al., 2008, and Dang et al., 2012). A study indicated that of MJ analogs for their antiinflammatory activities, the a-haloenones exhibited potent activity higher than those of natural anti-inflammatory prostaglandins (Dang et al., 2012). Methyl 4,5didehyrojasmonate also exhibited in vitro anti-inflammatory potency comparable to that of the most potent natural anti-inflammatory prostaglandin 15d-D. Among all these jasmonate analogs found that a-haloenone inhibited the production of pro-inflammatory mediators (nitric oxide (NO), interleukin (IL)1b, IL-6, and tumor necrosis factor (TNF)-a) through the down-regulation of NF-kB activity in lipo polysaccharide (LPS)activated murine macrophages.

\section{Antiparasitic activities}

Gold et al., (Gold et al., 2003) reported that jasmonates had significant antiparasitic effects on human parasites, Plasmodium falciparum (an intra erythrocytic protozoan parasite, which is the cause of the most severe form of malaria), and Schistosoma mansoni (a parasitic worm residing in blood vessels, causing a serious human infestation). Another case (Park et al., 2012) indicated that MJ induced a time- and dose-dependent decrease in the number of live Trichomonas vaginalis, an important human parasite of the urogenital tract. Other researchers (Ofer et al., 2008) also reported on the potential of employing MJ as a treatment for trichomoniasis, including its use against metronidazoleresistant strains.

In conclusion the recent years have been highly dynamic and productive for the field of JA research, with important discoveries emerging constantly. A large number of JA derivatives have been synthesized, isolated, and characterized. Jasmonate biosynthesis and functions has emerged which have potential to synthesis and functions of prostaglandins and leukotrienes in mammalians. Jasmonates are produced from a-linolenic acid from lipids which damaged plant membranes. The biosynthesis of these signalling compounds can be triggered through the activation of cell membrane receptors by pathogens, peptide signals, or wounding. These jasmonates are widely distributed in plants and affect a variety of processes namely as fruit ripening, production of viable pollen, root growth, and plant responses to wounding, abiotic stress, infections, and insects. Many of these new compounds have already been shown to have potent biological activity, promising that novel types of pharmacological agents possessing new mechanisms of action could soon become available. All these effects of JA are indirect, due to the well-studied induction of the biosynthesis of secondary compounds like anthocyanins, glucosinolates, terpenoids, or phenolics via transcription factors such as the Myb, Myc, and ORCA families. JA and its derivatives have emerged with medicinal, cosmetic, and flavoring uses and diverse applications in agriculture systems. During the last 20 years, the vast majority of studies and inventions have evidenced that JA, (b)-7iso-JA and MJ have anticancer activity against various cancers (e.g., prostate, lymphoid, breast, blood, skin cancers, etc). 


\section{References}

Aftab, T., Khan, M.M.A., Idrees, M., Naeem, M., Hashmi, N., 2011. Methyl jasmonate counteracts boron toxicity by preventing oxidative stress and regulating antioxidant enzyme activities and artemisinin biosynthesis in Artemisia annua L. Protoplasma, 248: 601-612

Browse, J., Howe, G.A. 2008. New weapons and a rapid response against insect attack. Plant Physiol. 146: 832-838.

Creelman, R.A., Mulpuri, R., 2002. The oxylipin pathway in Arabidopsis. The Arabidopsis Book 1. American Society of Plant Biologists, pp. e0012. doi:http:// dx.doi.org/10.1199/tab.0012.

Cao, S., Zheng, Y., Wang, K., Jin, P., Rui, H. 2009. Methyl jasmonate reduces chilling injury and enhances antioxidant enzyme activity in postharvest loquat fruit. Food Chem. 115: 1458-1463.

Carvajal, M., L. Espinoza, S. Caggia, V. Cardile, J.A. Garbarino, H., PeñaCortés. 2011. Electron. J. Biotechnol., 14: 15.

Dang, H.T., H.J. Lee, E. S. Yoo, B. Bao, J. S. Choi, J.H. Jung, Bioorg. Med. Chem., 16: 10228-10235.

Dang, H.T., Y.M. Lee, G.J. Kang, J. Hong, S.M. Lee, S.K. Lee, et al., Bioorg. Med. Chem., 20: 4109-4116.

Farmer, E.E., Ryan, C.A. 1990. Interplant communication: airborne methyl jasmonate induces synthesis of proteinase inhibitors in jasmonate signalling and ascorbate biosynthesis from plant leaves. Proc. Nat. Acad. Sci., 87: 7713-7716.

Farmer, E.E. 2007. Plant biology: jasmonate perception machines. Nature, 448: 659660.

Ferrari, S., Plotnikova, J.M., De Lorenzo, G., Ausubel, F.M., 2003. Arabidospis local resistance to Botrytis cinerea involves salicylic acid and camalexin and requires EDS4 and PAD2, but not SID2, EDS5 or PAD4. Plant J. 35, 193-205.

Gonzalez-Aguilar, G.A., Fortiz, J., Cruz, R., Baez, R., Wang, C.Y., 2000. Methyl jasmonate reduces chilling injury and maintains postharvest quality of mango fruit. J. Agri. Food Chem. 48: 515-519. Gonzalez-

Gold, D., I. Pankova-Kholmyansky, O. Fingrut, E. Flescher. 2003. J. Parasitol. 89: 1242-1244.

Gill, S.S., Anjum, N.A., Hasanuzzaman, M., Gill, R., Trivedi, D.K., Ahmad, I., Pereira, E., Tuteja, N. 2013. Glutathione and glutathione reductase: a boon in disguise for plant abiotic stress defense operations. Plant Physiol. Biochem., 70: 204-212.

Gunes, A., Inal, A., Bagci, E.G., Coban, S., Sahin, O. 2007. Silicon increases B tolerance and reduces oxidative damage of wheat grown in soil with excess B. Biol. Plant., 51: 571-574.

Gfeller, A., Liechti, R., Farmer, E.E., 2010. Arabidopsis jasmonate signaling pathway. Sci. Signal. 3 doi: http://dx.doi.org/10.1126/scisignal.3109 cm4.

Hamberg, M., Gardner, H.W. 1992. Oxylipin pathway to jasmonates: biochemistry and biological significance. Biochim. Biophys. Acta, 1165: 1-18.

Halitschke, R., Baldwin, I.T. 2004. Jasmonates and related compounds in plantinsect interactions. J. Plant Growth Regul., 23: 238-245.

Inal, A., Pilbeam, D.J., Gunes, A. 2009. Silicon increases tolerance to B toxicity and reduces oxidative damage in barley. J. Plant Nutr., 32: 112-128.

James, D.G., 2005. Further field evaluation of synthetic herbivore-induced plan volatiles as attractants for beneficial insects. J. Chem. Ecol., 31: 481-495.

Khan, M.I.R., Iqbal, N., Masood, A., Per, 
T.S., Khan, N.A. 2013. Salicylic acid alleviates adverse effects of heat stress on photosynthesis through changes in proline production and ethylene formation. Plant Signal. Behav., 8: 263274.

Koda, Y. 1992. The role of jasmonic acid and related compounds in the regulation of plant development. Int. Rev. Cytol., 135: 155-199.

Lin, T., Sharma, P., Gonzalez, D.H., Viola, I.L., Hannapel, D.J., 2013. The impact of the long-distance transport of a BEL1-like messenger RNA on development. Plant Physiol. 161: 760 772 .

Michelet, J.F., Olive, C., Rieux, E., Fagot, D., Simonett, L., Galey, J.F., Dalko-Csiba, M., Bernard, B.A., Pereira, R. 2012. The anti-ageing potential of a new jasmonic acid derivative (LR2412): in vitro evaluation using reconstructed epidermis episkin TM. Exp. Dermatol. 21, 390-400.

Masood, A., Iqbal, N., Khan, N.A. 2012. Role of ethylene in alleviation of cadmium induced photosynthetic capacity inhibition by sulphur in mustard. Plant Cell Environ., 35; 524-533.

Meir, S., Droby, S., Davidson, H., Alsevia, S., Cohen, L., Horev, B., Philosoph-Hadas, S. 1998. Suppression of Botrytis rot in cut rose flowers by postharvest application of methyl jasmonate. Postharvest Biol. Technol., 13: 235243.

Noctor, G., Foyer, C.H. 1997. Simultaneous measurement of foliar glutathione, gglutamylcysteine, and amino acids by high-performance liquid chromatography Comparison with two other assay methods for glutathione. Anal. Biochem., 264, 98-110.

Ofer, K., D. Gold, E. Flescher, Int. J. Parasitol., 38: 959-968.

Paull, R.E., Rohrbach, K.G. 1985. Symptom development of chilling injury in pineapple fruit (Ananascomosus). $J$. Am. Soc. Hort. Sci., 110, 100-105.

Paull, J.G., Nable, R.O., Rathjen, A.J., 1992. Physiological and genetic control of the tolerance of wheat to high concentrations of B and implications for plant breeding. Plant Soil, 146: 251260.

Pedranzani, H., Racagni, G., Alemano, S., Miersch, O., Ramirez, I., Pena-Cortes, H., Taleisnik, E., Machado-Domenech, E., Abdala, G., 2003. Salt tolerant tomato plants show increased levels of jasmonic acid. Plant Growth Reg., 41: 149-158.

Park, C., C.Y. Jin, H.J. Hwang, F. Flescher. 2012. Toxicol. In Vitro, 26: 86 -93.

Reinbothe, C., Springer, A., Samol, I., Reinbothe, S. 2009. Plant oxylipins: role of jasmonic acid during programmed cell death, defence and leaf senescence. F.E. B.S. J., 276: 4666-4681.

Soriano, I.R., Asenstorfer, R.E., Schmidt, O., Riley, I.T. 2004. Inducible flavone in oats (Avena sativa) is a novel defense against plant-parasitic nematodes. Phytopathol., 94: 1207-1214.

Takahashi, H., Kanayama, Y., Zheng, M.S., Kusano, T., Hase, S., Ikegami, M., Shah, J., 2004. Antagonistic Interactions between the SA and JA signaling pathways in Arabidopsis modulate expression of defense genes and genefor-gene resistance to cucumber mosaic virus. Plant Cell Physiol., 45, 803-809.

Wasternack, C., 2007. Jasmonates: an update on biosynthesis, signal transduction and action in plant stress response, growth and development. Ann. Bot., 100, 681697.

Wasternack, C. 2014. Action of jasmonates in plant stress responses and developmentapplied aspects. Biotechnol. Adv., 32: 31-39. 
Wasternack, C., Hause, B., 2002. Jasmonates and octadecanoids: signals in plant stress responses and development. Prog. Nucleic Acid Res. Mol. Biol., 72: 165221.

Wasternack, C., Hause, B. 2013. Jasmonates: biosynthesis, perception, signal transduction and action in plant stress response, growth and development. An update to the 2007 review in Annals of Botany. Ann. Bot. 111, 1021-1058. Weber, H., 2002. Fatty acid-derived signals in plants. Trends Plant Sci., 7: 217-224.

Wang, C.Y., Buta, G. 1994. Methyl jasmonate reduces chilling injury in Curcubita pepo through its regulation of abscisic and polyamine levels. Environ. Exp. Bot., 43: 427-432.
Xue, R., Zhang, B. 2007. Increased endogenous methyl jasmonate altered leaf and root development in transgenic soybean plants. J. Genet. Genomics, 34: 339-346.

Zhang, J., Mu, C. 2009. Effects of saline and alkaline stresses on the germination growth, photosynthesis, ionic balance and anti-oxidant system in an alkalitolerant leguminous forage Lathyrus quinquenervius. Soil Sci. Plant Nut., 55: 685-697.

Zhou, M.L., Yang, X.B., Zhang, Q., Zhou, M., Zhao, E.Z., Tang, Y.X., Zhu, X.M., Shao, J.R., Wu, Y.M. 2013. Induction of annexin by heavy metals and jasmonic acid in Zea mays. Funct. Integr. Genomics, 13: 241-251.

\section{How to cite this article:}

Samota, M.K., L. Bhatt, N. Garg and Geat, N. 2017. Defense Induced by Jasmonic Acid: A Review. Int.J.Curr.Microbiol.App.Sci. 6(5): 2467-2474. doi: https://doi.org/10.20546/ijcmas.2017.605.276 